\title{
SLOTDEEP: A DOS COMPUTER PROGRAM FOR C(ORRELATING PALYNOLOGY SITES USING THE VISUAL INFORMATION IN AN EXPLODED MATRIX OF THEIR DISSIMILARITY COEFFICIENTS
}

MAHER, Louis J., Jr., Department of Geology and Geophysics, University of Wisconsin, 1215 W. Dayton St., Madison, WI 53706, USA. E-mail: maher@geology.wisc.edu

At the end of a paleoecological investigation, the results have to be correlated with other sites to see how the new information ties in with the old. The standard pollen zonation schemes of Europe and North America emphasize that the sequential changes in taxon composition in a single core are mirrored in other cores of the region. Anyone can recognize pollen zones when they are pointed out on a diagram, but almost no two workers will agree exactly where one zone ends and another begins. This sort of problem can be explored by numerical analysis: where can a sequence be divided that will yield two adjacent parts that are most alike in themselves and most unalike between themselves? The zone boundaries are the datable entities, and this explains the efforts to define them. Sites also can be correlated by constructing a matrix of dissimilarity coefficients (DC) between their sample levels and letting a dynamic algorithm calculate a "minimum-cost" route through the matrix that will "slot" or intercalate the two sites. However there is a paradox involved when correlating by pollen zonation and correlating by slotting. Pollen zone boundaries are potential points of correlation; pollen zones often prevent effective slotting. SLOTDEEP is a computer program that helps deal with the paradox.

SLOTDEEP works on DOS computers and gives the user a powerful tool for correlating pollen cores. Utilizing raw pollen counts from two sites, the program processes the data and shows their abridged pollen diagrams simultaneously on the screen. The program also calculates a DC matrix - with a choice of several different types-between all the levels of both sites. The program uses a standard technique to slot the sites automatically, and allows the user to see the color-coded results on a combined pollen diagram. But more important, the user also has the opportunity to see the DC matrix on the screen, coded by color and separated (exploded) according to each core's sample spacing -information normally lost when slotting matrices. The human brain is well-suited for recognizing patterns in such displays. The program allows one to use these spacial patterns in the correlation by moving the cursor to manually anchor good matches on the matrix. Getting information from a picture of the matrix allows one to examine the data from a perspective not normally available. The result of this correlation can be viewed as a slotted and color-coded standard diagram; when the operator is satisfied, the correlation can be saved to a disk file. The operator can also convert the depth/lime data of the secondary site to its equivalent in the principal site. Allowing the human to do what he/she does well, often provides a much more convincing correlation then by relying on the computer alone.

SLOTDEEP, auxiliary programs, and sample data are available by Worldwide Web. The URL is: http://geology.wisc.edu/ maher/inqua.html and the programs are free. 